\title{
Some theoretical models for solitary structures of boundary layer
}

\section{waves}

\author{
G. S. Lakhina ${ }^{1}$, B. T. Tsurutani ${ }^{2}$, S. V. Singh ${ }^{3}$, and R. V. Reddy ${ }^{3}$ \\ ${ }^{1}$ Indian Institute of Geomagnetism, Colaba, Mumbai, India \\ ${ }^{2}$ Jet Propulsion Laboratory, California Institute of Technology, Pasadena, California, USA \\ ${ }^{3}$ Indian Institute of Geomagnetism, Colaba, Mumbai, India
}

Received: 26 December 2001 - Accepted: 5 March 2002

\begin{abstract}
Solitary electrostatic structures have been observed in and around auroral zone field lines at various altitudes ranging from close to the ionosphere, to the magnetopause low-latitude boundary layer (LLBL) and cusp on the dayside, and to the plasma sheet boundary layer on the nightside. In this review, various models based on solitons/double layers and BGK modes or phase space holes are discussed in order to explain the characteristics of these solitary structures.
\end{abstract}

\section{Introduction}

Plasma wave data from the Plasma Wave Instrument (PWI) on Polar spacecraft indicate the presence of intense broadband plasma waves on the polar cap magnetic field lines which map to the low-latitude (magnetopause) boundary layer (LLBL). These waves are in the range of ELF/VLF frequencies, are spiky and their frequency dependence and intensities are quite similar to those of magnetopause boundary layer waves (Gurnett et al., 1979; Tsurutani et al., 1981, 1989; Anderson et al., 1982; Gendrin, 1983; LaBelle and Treumann, 1988; Rezeau et al., 1986; Zhu et al., 1996; Song et al., 1998). These waves, therefore, are called polar cap boundary layer (PCBL) waves (Tsurutani et al., 1998a). The high-time resolution plasma wave data from Polar, in the polar cap boundary layer (high-altitude auroral zone field lines), have resolved the question about the cause of the "broad bandedness" of the PCBL wave (Franz et al., 1998; Tsurutani et al., 1998b). There are indeed whistler mode emissions present, as suggested by Gurnett et al. (1979), but the waves are patchy packets lasting only $\sim 10 \mathrm{~ms}$ in duration. The packets occur with a variety of frequencies, and when integrated over time, a rough power-law power spectrum is formed. Superposed with the whistler mode emissions are electric solitary waves with $\sim$ ms durations (Franz et al., 1998; Tsurutani et al., 1998b; Lakhina and Tsurutani, 1999).

Correspondence to: G. S. Lakhina (lakhina@iig.iigm.res.in)
Such solitary waves (bipolar pulse structures) have been observed earlier by the S3-3 (Temerin et al., 1982) and the Viking (Boström et al., 1988; Koskinen et al., 1990) spacecraft, and recently by FAST (Ergun et al., 1998; McFadden et al., 1999). Further, from the analysis of Polar perigee data at auroral altitudes $\sim 6000 \mathrm{~km}$, Mozer et al. (1997) found that such solitary structures were associated with hydrogen ion cyclotron waves. Matsumoto et al. (1994) and Kojima et al. (1997) have detected them in the near-Earth plasma sheet boundary layer on board GEOTAIL.

The typical scale sizes, $L_{\|}$, and velocities, $V_{o \|}$, of solitary structures parallel to the ambient magnetic field, as deduced from the Polar data, are $L_{\|} \sim 100-1000 \mathrm{~m}$, $V_{o \| \mid} \sim 1000 \mathrm{~km} \mathrm{~s}^{-1}$ or higher (Franz et al., 1998). For the case of GEOTAIL, $L_{\|} \sim 2-5 \mathrm{~km}$ and $V_{o \|} \sim 1000 \mathrm{~km} \mathrm{~s}^{-1}$ and higher (Matsumoto et al., 1994; Kojima et al., 1997), whereas the FAST data indicate $L_{\|} \sim$ a few Debye lengths and $V o_{\|} \gg C_{s}$, where $C_{s}$ is the ion acoustic speed (Ergun et al., 1998). Franz et al. (2000) have shown that electron holes observed by Polar are roughly spherical for $f_{c e} / f_{p e}>1$, becoming more oblate (with perpendicular scale larger than the parallel scale) with decreasing $f_{c e} / f_{p e}$. Here, $f_{c e}$ and $f_{p e}$ represent, respectively, the cyclotron and plasma frequency of the electron. A comparison of the characteristics of solitary waves, such as their scale sizes and velocities, observed by Polar in the high-latitude cusp, polar cap and plasma sheet boundary layer, have been given by Cattell et al. (1999).

\section{Theoretical models}

Various models have been proposed to explain the solitary pulses (for a review, Lakhina et al., 2000a). The bipolar electric structures observed by Polar and FAST have positive potentials and are speculated to be electron holes (Mozer et al., 1997; Franz et al., 1998; Tsurutani et al., 1998b; Ergun et al., 1998a, b; Goldman et al., 1999; Muschetti et al., 1999). The solitary structures observed by S3-3 and Viking have negative potentials and are interpreted as ion solitary structures 
or ion holes (Temerin et al., 1982; Boström et al. 1988; Gray et al., 1991). Pottelette et al. (1990) and Dubouloz et al. (1991a) showed theoretically that the generation of broad band electrostatic noise can be interpreted in terms of traveling electron-acoustic solitons. Bounds et al. (1999) have found that positively potential solitary structures (electron holes) are associated with electron beams, whereas the ion solitary structures are associated with ion beams. Therefore, all models fall basically into two categories, namely a) the soliton/solitary wave models, and b) the BGK/Phase space holes models.

\subsection{Solitons/solitary waves}

\subsubsection{Ion acoustic solitons and double layers}

Solitons (double layers) are the localized symmetric (asymmetric) potential structures with no net potential drop (a net potential drop). Such localized structures were detected on the auroral field lines in the altitude range of 6000 to $12000 \mathrm{~km}$ for the first time by S3-3 (Temerin et al., 1982) and later on by Viking (Boström et al., 1988; Koskinen et al., 1990). Several attempts have been made to explain these observations in terms of ion acoustic solitary waves (Buti, 1980; Yu et al., 1980; Hudson et al., 1983; Qian et al., 1988; Reddy and Lakhina, 1991; Reddy et al., 1992). Bharutram and Shukla (1985) considered multidimensional solitons and double layers in a magnetized plasma by taking into account the Poisson equation, rather than the quasi-neutrality condition. Berthomier et al. (1998) studied the characteristics of ion acoustic solitary waves and weak double layers in twoelectron temperature auroral plasma. The auroral plasma in the auroral acceleration region is characterized by multi-ion beams, such as $\mathrm{H}^{+}, \mathrm{O}^{+}, \mathrm{He}^{+}$and a two-electron population. Reddy et al. (1992) developed a general analysis for small amplitude ion-acoustic solitons and double layers that take into account any number of ion beams and their charges, together with cold and hot electrons. For the auroral plasma parameters with only $\mathrm{H}^{+}-\mathrm{O}^{+}$beams, their analysis predict the excitation of fast and slow hydrogen (as well oxygen) beam acoustic modes, which can be either rarefactive double layers, rarefactive, or compressive solitons.

Figure 1 shows the variation of the potential $\Psi$ versus the distance-like parameter $\eta$ for the auroral plasma with twoion species, i.e. $\mathrm{H}^{+}$and $\mathrm{O}^{+}$, for a fixed value of oxygen beam density $\mathrm{N}_{\mathrm{O}+}$, hydrogen ion beam density $\mathrm{N}_{\mathrm{H}+}$, and the temperature ratio of hot to cool electron components, $T_{h} / T_{c}$. In Fig. 1a, oxygen beam speed $U_{\mathrm{O}+}$, hydrogen beam speed $U_{\mathrm{H}+}$, and the temperature of oxygen beam $T_{\mathrm{O}+}$, are held constant while the temperature of the hydrogen beam $T_{\mathrm{H}+}$ is varied. Here, all speeds are normalized with respect to the oxygen ion acoustic speed, $C_{s o}=\left(T_{e f f} / m_{o}\right)^{1 / 2}$; densities are normalized with respect to total electron density $n_{o}=n_{c}+n_{h}$ and the temperatures are normalized with respect to the effective temperature of the electrons, $T_{e f f}=n_{o} T_{c} T_{h} /\left(n_{c} T_{h}+n_{h} T_{c}\right)$, where $n_{c}\left(n_{h}\right)$ and $T_{c}\left(T_{h}\right)$ are the density and temperature of the cold (hot) electrons. For
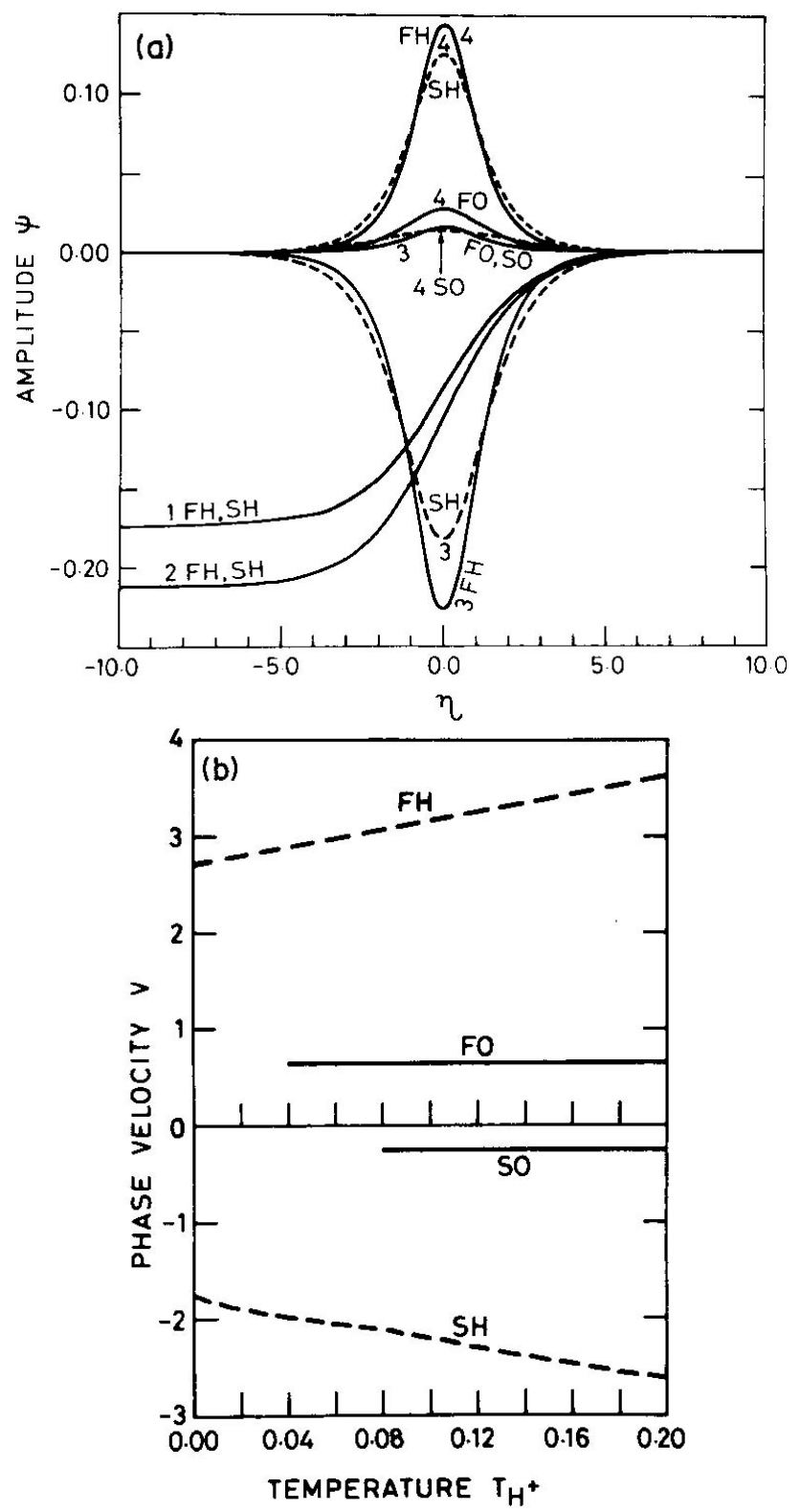

Fig. 1. (a) Double layer and soliton solutions for the auroral plasma parameters $n_{\mathrm{O}+}=0.4 n_{0}$ (where $n_{0}$ is the total electron density in the equilibrium state), $Z_{2}\left(=m_{O} / m_{H}\right)=16.0, n_{h}=0.9 n_{0}, M=$ $0.1, \alpha=0.01, T_{h} / T_{c}=500.0, U_{\mathrm{O}+}=0.2, U_{\mathrm{H}+}=0.5$ and $T_{\mathrm{O}+}=0.1$. The curves $1-4$ correspond to the case of $T_{\mathrm{H}+}=$ $0.0,0.02,0.10$ and 0.2 , respectively. The curves 1 and 2 represent rarefactive double layers. Curves $3(\mathrm{FH}), 3(\mathrm{SH})$ represent rarefactive solitons and curve 4 represents a compressive soliton. Here, the notation $\mathrm{FH}, \mathrm{SH}, \mathrm{FO}$ and $\mathrm{SO}$ stands for fast hydrogen, slow hydrogen, fast oxygen and slow oxygen beam-acoustic modes, respectively. (b) Phase velocity $V$ vs. temperature $T_{\mathrm{H}+}$ hydrogen beam for admissible solutions of Eqs. (24) and (26) of Reddy et al. (1992). The other plasma parameters are the same as in (a) (taken from Reddy et al., 1992).

both fast and slow hydrogen acoustic modes, denoted by $\mathrm{FH}$ and $\mathrm{SH}$, it is seen that as $T_{\mathrm{H}+}$ increases, the rarefactive double layer (RDL) becomes converted to the rarefactive soli- 


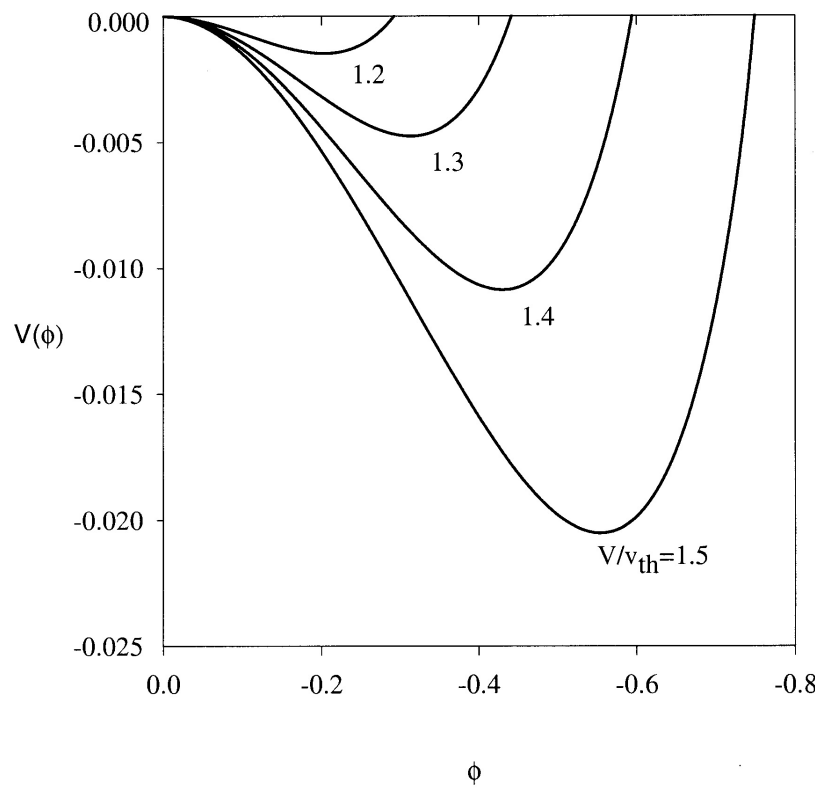

Fig. 2. Sagdeev potential $V(\phi)$ vs. potential $\phi$ for $n_{o c}=0.2 \mathrm{~cm}^{-3}$, $n_{o h}=1.5 \mathrm{~cm}^{-3}, n_{o b}=1.0 \mathrm{~cm}^{-3}, T_{c} / T_{h}=0.001, T_{i} / T_{h}=$ 0.01 , and $v_{o} / V_{t h}=0.1$ for various values of normalized soliton velocity $V / V_{t h}$, as indicated on the curves (taken from Singh et al., 2001a).

ton (RS) and then to compressive soliton (CS). The strength and width of the RDL increases with the increasing temperature of the hydrogen beam. The slow and fast oxygenacoustic modes, labeled SO and FO, respectively, are significant only at $T_{\mathrm{H}+}=0.1$ and 0.2 . Figure $1 \mathrm{~b}$ shows the variation of the normalized phase velocity, $V\left(=V_{p h} / C_{s o}\right)$, with $T_{\mathrm{H}+}$. It is seen that phase velocities of both fast $(\mathrm{FH})$ and slow (SH) hydrogen-acoustic modes increase with increasing $T_{\mathrm{H}+}$, whereas that of fast (FO) and slow (SO) oxygenacoustic modes remain nearly constant.

The analysis of Reddy et al. (1992) predicts the typical width of the double layers as $W_{D L} \sim(5-8) \rho_{o} \sim(600-$ 960) $\mathrm{m}$ and that of solitons, $W_{s} \simeq(2-5) \rho_{o} \sim(240-600) \mathrm{m}$, where $\rho_{o}$ is the gyroradius of oxygen ions. In the auroral acceleration region, $\rho_{o} \sim 120 \mathrm{~m}$. The observed widths of the solitary structures by S3-3 and Viking are $\sim 200 \mathrm{~m}$ (Koskinen et al., 1990; Temerin et al., 1982). Further, the phase velocities of DL/solitons are typically $V_{p h} \simeq(0.25-20) C_{s o} \simeq$ $(2-150) \mathrm{km} \mathrm{s}^{-1}$. The observed velocities of the solitary structures are $\simeq 50 \mathrm{~km} \mathrm{~s}^{-1}$.

\subsubsection{Electron acoustic solitons}

A detailed study of the properties of the broad band electrostatic noise in the dayside auroral zone has been presented by Dubouloz et al. (1991b). Dubouloz et al. (1991a, 1993) studied the electron-acoustic solitons (EAS) with two electroncomponents (cold and hot) and motionless ions, and showed that the high-frequency broad band electrostatic noise in the dayside auroral zone can be generated by the EAS. The effect of upward propagating electron beams that are commonly observed in the dayside auroral zone was not taken into consideration. Berthomier et al. (2000) performed a parametric study of the small amplitude electron-acoustic solitons in an electron-beam plasma system.

Recently, Singh et al. (2001a) have extended the model of Dubouloz et al. (1991a) by including the electron beam dynamics, ion motion, and adiabatic equation of state. They studied arbitrary amplitude electron-acoustic solitons in an unmagnetized auroral plasma with four components, namely non-drifting Maxwellian hot electrons and fluid cold electrons, an electron beam drifting along the $x$-axis, the direction of propagation of the electron-acoustic waves, and fluid ions. In a stationary frame moving with speed $V$, the set of fluid equations and the Poisson equation, on applying appropriate boundary conditions, can be reduced in the form of an energy equation

$\frac{1}{2}\left(\frac{d \phi}{d \xi}\right)^{2}+V(\phi)=0$,

where $\phi$ is the normalized potential of the nonlinear electron acoustic wave, $\xi$ is the normalized distance variable in the stationary frame, and $V(\phi)$ is the Sagdeev potential which is a complicated function of $\phi$, as well as plasma parameters (Singh et al., 2001a). The soliton solutions of Eq. (1) exist when the usual conditions $V(\phi)=0$ and $d V(\phi) / d \phi=0$ at $\phi=0$ and $V(\phi)<0$ for $0<|\phi|<\left|\phi_{0}\right|$ are satisfied, where $\phi_{0}$ is the maximum amplitude of the solitons. Figure 2 shows the variation of Sagdeev potential $V(\phi)$ with the potential $\phi$ for the auroral region parameters for an event observed by Viking (Dubouloz et al., 1993). It is seen that electron acoustic solitons can exist for $1<V / v_{t h}<1.5$, where $v_{t h}$ is the thermal velocity of the hot electron component.

Figure 3 shows the variation of soliton amplitude $\phi$ with $\xi$ for the parameters of Fig. 2 for different values of normalized electron beam drift velocity, $v_{0} / v_{t h}$. The soliton amplitude decreases with the increase in the drift velocity and the soliton solutions do not exist for $v_{0} / v_{t h}>0.5$. Also, small values of the temperature ratio of beam to hot electrons, $T_{b} / T_{h}$, are required, in order to obtain soliton solutions (not shown). Further, it is found that soliton solutions exist only when the hot electron density, $n_{0 h}$, is in the range of $0.75 \mathrm{~cm}^{-3}<n_{0 h}<1.65 \mathrm{~cm}^{-3}$. The soliton amplitude is found to increase with the increase in the hot electron density within this range.

For the typical parameters, namely cold electron density $n_{0 c}=0.2 \mathrm{~cm}^{3}$, hot electron density $n_{0 h}=1.5 \mathrm{~cm}^{-3}$, beam electron density $n_{0 b}=1.0 \mathrm{~cm}^{-3}, T_{h}=100 \mathrm{eV}, T_{b} \sim 1 \mathrm{eV}$, $T_{c}=T_{i} \sim 1 \mathrm{eV}$, and $v_{0} / v_{t h}=0.01-0.5$, i.e. corresponding to the electron beam drift velocity $v_{0} \sim 40-2100 \mathrm{~km} \mathrm{~s}^{-1}$, the typical electric field associated with solitons is found to be $E_{\|} \sim 10-400 \mathrm{mV} / \mathrm{m}$ and the typical soliton half widths are $\sim 1-4$ Debye in length. The typical velocities of the electron-acoustic solitary structures are found to be $\sim(1.1-1.5) v_{t h} \sim(4500-6300) \mathrm{km} \mathrm{s}^{-1}$. These predictions of the model need to be checked with the observed beam parameters in the auroral acceleration region. 


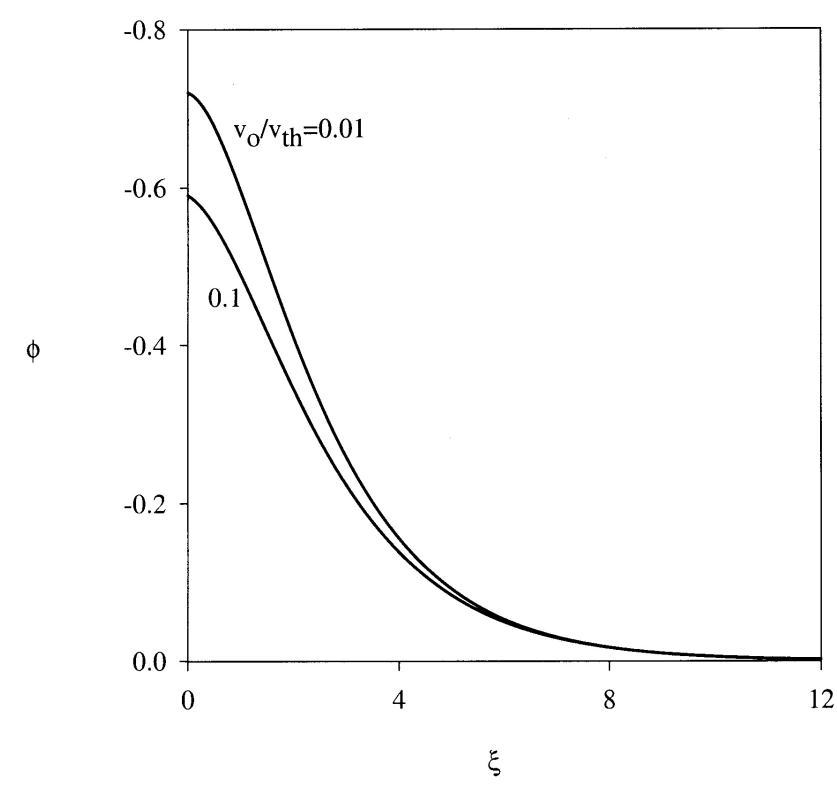

Fig. 3. Potential $\phi$ vs. $\xi$ for various values of $v_{o} / V_{t h}$ for $V / V_{t h}=$ 1.4. Other parameters are the same as Fig. 2 (taken from Singh et al., 2001a).

Recently, Ghosh et al. (2000) have shown that nonlinear evolution of 2-D electron-acoustic waves is governed by the Davey-Stewartson I equation, which contains so-called dromion solutions. It is shown that increasing electron temperature enlarges the dromion's parametric space. Implications of these two-dimensional localized solutions to the observed solitary structures needs to be explored.

\subsubsection{Nonlinear ion cyclotron waves}

Reddy et al. (2002) have developed an analytical model of the coupled nonlinear ion cyclotron and ion-acoustic waves, which could explain the FAST observations (Ergun et al., 1998) of strong, spiky waveforms in the parallel electric field, in association with ion cyclotron oscillations in the perpendicular electric fields. Using the fluid equations for the plasma consisting of warm electrons and cold ions, a nonlinear wave equation is derived in the rest frame of the propagating wave for any direction of propagation oblique to the ambient magnetic field. The equilibrium bulk flow of ions, $v_{0}$, is also included in the model to mimic the field-aligned current. In a frame of reference, where the space coordinate $x$ and time coordinate $t$ depend on a single variable $\eta=(x-V t)\left(\Omega_{i} / V\right)$, where $\Omega_{i}$ is the ion cyclotron frequency and $V$ is the phase velocity of the wave, the evolution equation for the normalized potential $\Psi$ of the coupled nonlinear ion cyclotron and ion-acoustic waves can be written as

$$
\left(e^{-2 \Psi}-M_{A}^{-2}\right) \frac{d^{2} \Psi}{d \eta^{2}}-2 e^{-2 \Psi}\left(\frac{d \Psi}{d \eta}\right)^{2}+\chi(\Psi)=0
$$

where

$$
\begin{gathered}
\chi(\Psi)=e^{\Psi}\left(\frac{M^{2}}{M_{A}^{2}}+2 E_{0}^{2}\right)-\frac{M^{2}}{M_{A}^{2}}- \\
\frac{M^{2} \cos ^{2} \theta e^{\Psi}}{M_{A}^{2}}\left(e^{\Psi}-1\right) .
\end{gathered}
$$

Equation (2) is derived using the conditions, $\Psi=$ $0, d \Psi / d \eta=E_{0}$, and $d^{2} \Psi / d \eta^{2}=0$ at $\eta=0$. Here, $M_{A}=\left(M-v_{0} / C_{s}\right)$, where $C_{s}$ is the ion acoustic speed, $M=V / C_{s}$ is the Mach number of the nonlinear wave, and $E_{0}$ is the driving amplitude of the electric field at $\eta=0$. For $E_{0}=0, v_{0}=0$, and $\theta=90^{\circ}$, Eq. (2) reduces to the evolution equation of the perpendicularly propagating wave (Temerin et al., 1979). For $E_{0}=0, v_{0}=0$, Eq. (2) goes over to that of Lee and Kan (1981).

Equation (2) has been solved numerically by Reddy et al. (2002) over a wide parametric space. For nearly parallel propagation, the electric field is found to exhibit a variety of waveforms, for example, sinusoidal, sawtooth or highly spiky waveforms, depending upon the driving amplitude $E_{0}$, Mach number $M$, and the bulk flow velocity $v_{0}$. Figure 4 shows the variation of the parallel electric field for Mach number, $M=1.25, v_{0}=0$ and for different driver strengths. It is seen that the $E_{\|}$shows ion cyclotron oscillations for small $E_{0}$ (Fig. 4a) and with increasing driver strength, the cyclotron oscillations tend to steepen (Fig. 4b). As the driving amplitude increases, a driven ion acoustic mode, which exhibits waveforms somewhat similar to the sawtooth type with a period twice the ion cyclotron period, is seen in Fig. 4c. A further increase in driver strength yields a driven ion acoustic oscillation with highly spiky bipolar structure in the waveform, with a periodicity of about four times the ion cyclotron period (Fig. 4d) and with a great deal of similarity to the FAST observation (Ergun et al., 1998). Using the actual number for various parameters, the spiky structures are found to have $E_{\|} \sim 50 \mathrm{~m} \mathrm{~V} / \mathrm{m}$ to a few $\mathrm{V} / \mathrm{m}$, scale sizes $\sim(75-1500) \mathrm{m}$ and velocities $\sim(10-600) \mathrm{km} \mathrm{s}^{-1}$. Observations by FAST can be explained naturally by assuming a mixture of near-parallel and near-perpendicular propagating wave modes.

\subsection{BGK modes/phase space holes}

These models are based on the solutions of the stationary Vlasov equation and Poisson's equation. The trapped particles play crucial roles in these models (Bernstein et al., 1957; Turikov, 1984). The waveform observations by the plasma wave instrument on board the Geotail spacecraft have shown that broad band electrostatic noise (BEN) consists of a series of bipolar solitary pulses (Matsumoto et al., 1994). They showed that the broadness of the BEN frequency spectra arises from the solitary waveforms. A likely generation mechanism for BEN is based on the nonlinear evolution of the electron beam instabilities, leading to the formation of the isolated Bernstein-Greene-Kruskal (BGK) potential structures, which reproduce well the observed electrostatic 


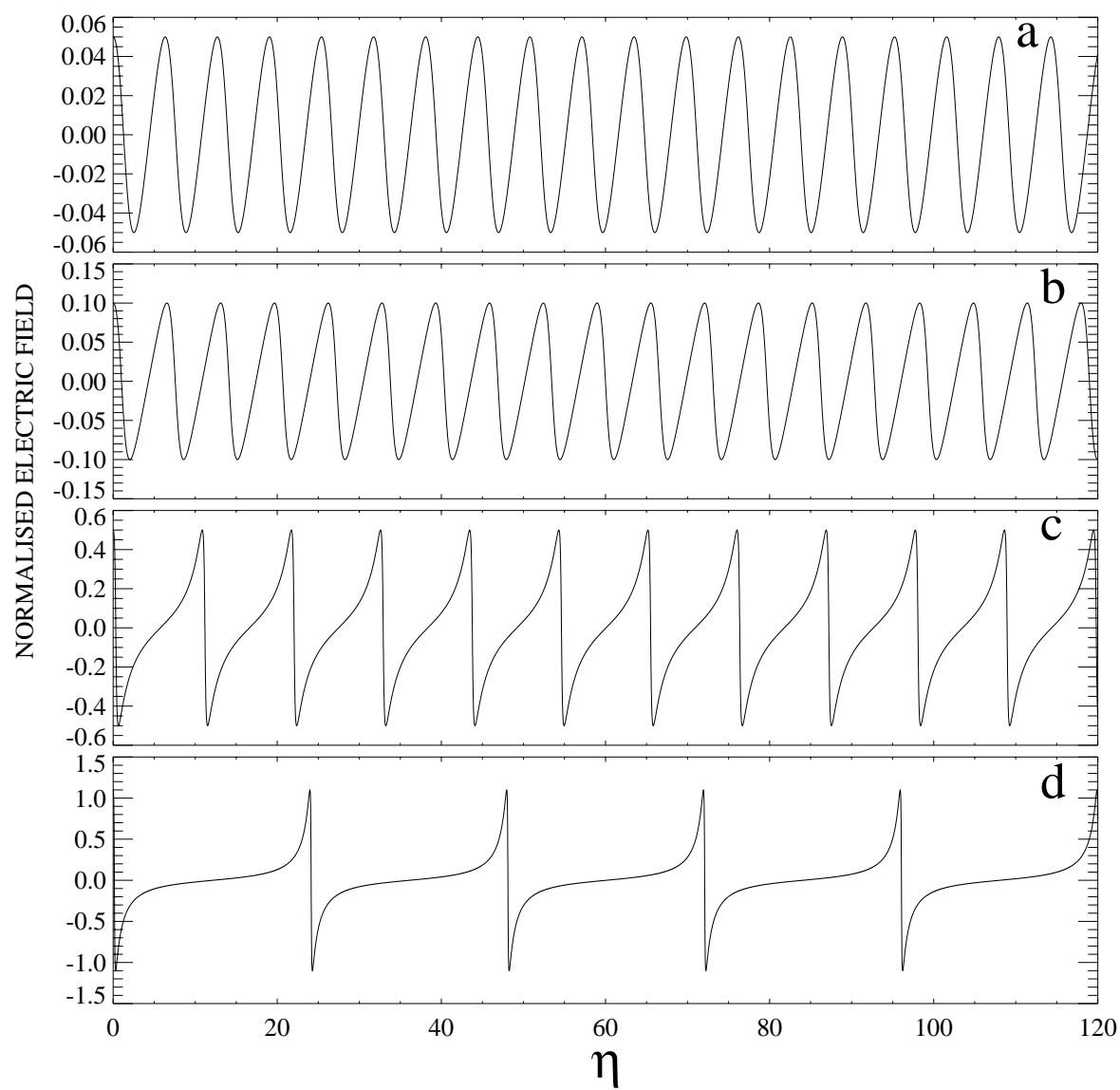

Fig. 4. Numerical solution of the normalized parallel electric field $(=\partial \Psi / \partial \eta)$ of nonlinear electrostatic ion cyclotron and ion acoustic waves for the parameters $\theta=2^{0}, M=1.25, \delta=0$ and $E_{0}=0.05$ (a), 0.10 (b), 0.5 (c), and 1.1 (d) (taken from Reddy et al., 2002).

solitary waveforms (Omura et al., 1994, 1996, 1999; Kojima et al., 1997, 1999). An example of the time evolution of the bump-on-tail instability obtained by the one-dimensional computer simulations is shown in Fig. 5. The right and left panels display the time series of electron phase diagrams and reduced electron velocity distributions. The vortices shown in the left panel are electron holes formed by trapped electrons due to isolated electrostatic potentials, which are generated by the nonlinear evolution of the bump-on-tail instability.

Muschietti et al. (1999) have interpreted these solitary structures observed by FAST (Ergun et al., 1998a, b) in terms of BGK phase-space electron holes drifting along the magnetic field lines. Goldman et al. $(1999,2000)$ have given an explanation for these bipolar structures in terms of nonlinear two-stream instabilities, a mechanism similar to that of the plasma sheet boundary layer BEN proposed by Omura et al. (1996) and Kojima et al. (1997). An example of the 2-D simulations of counterstreaming electron beam instability done by Goldman et al. (2000) is shown in Fig. 6. It is seen that the initial evolution to bipolar structures in the $x$ direction, the direction of the ambient magnetic field, is followed by the excitation of whistlers, which dominate at later times.
Singh et al. (2001b) have performed 3-D particle simulation of electron beam driven instabilities to explain the characteristics of the solitary structures observed on the auroral field lines. Figure 7 shows some results of their simulations for a finite-sized fast electron beam with $V_{b} / V_{t e}=8$. Here, $V_{b}$ is the beam velocity and $V_{t e}$ is the electron thermal speed. It is seen that the high-frequency plasma waves are generated first, followed by the dominance of lower hybrid (LH) waves, then ion cyclotron waves (see left-hand panel). The electron hole vortexes form, but they are transitory, as seen from the right-hand panel. On the other hand, simulations with relatively slow electron beams show the formation of long-lasting electron holes, especially when the electrons are highly magnetized (Singh et al., 2001c). Both simulations indicate ion heating due to lower hybrid waves.

\section{Conclusions}

The solitary structures observed by several spacecraft in different regions of the magnetosphere, for example, auroral zone field lines, including the polar cap boundary layer, cusp, and the plasma sheet boundary layer, can be explained either in terms of soliton models or BGK modes, where trapping of electrons in the nonlinear wave plays a crucial role. The soli- 

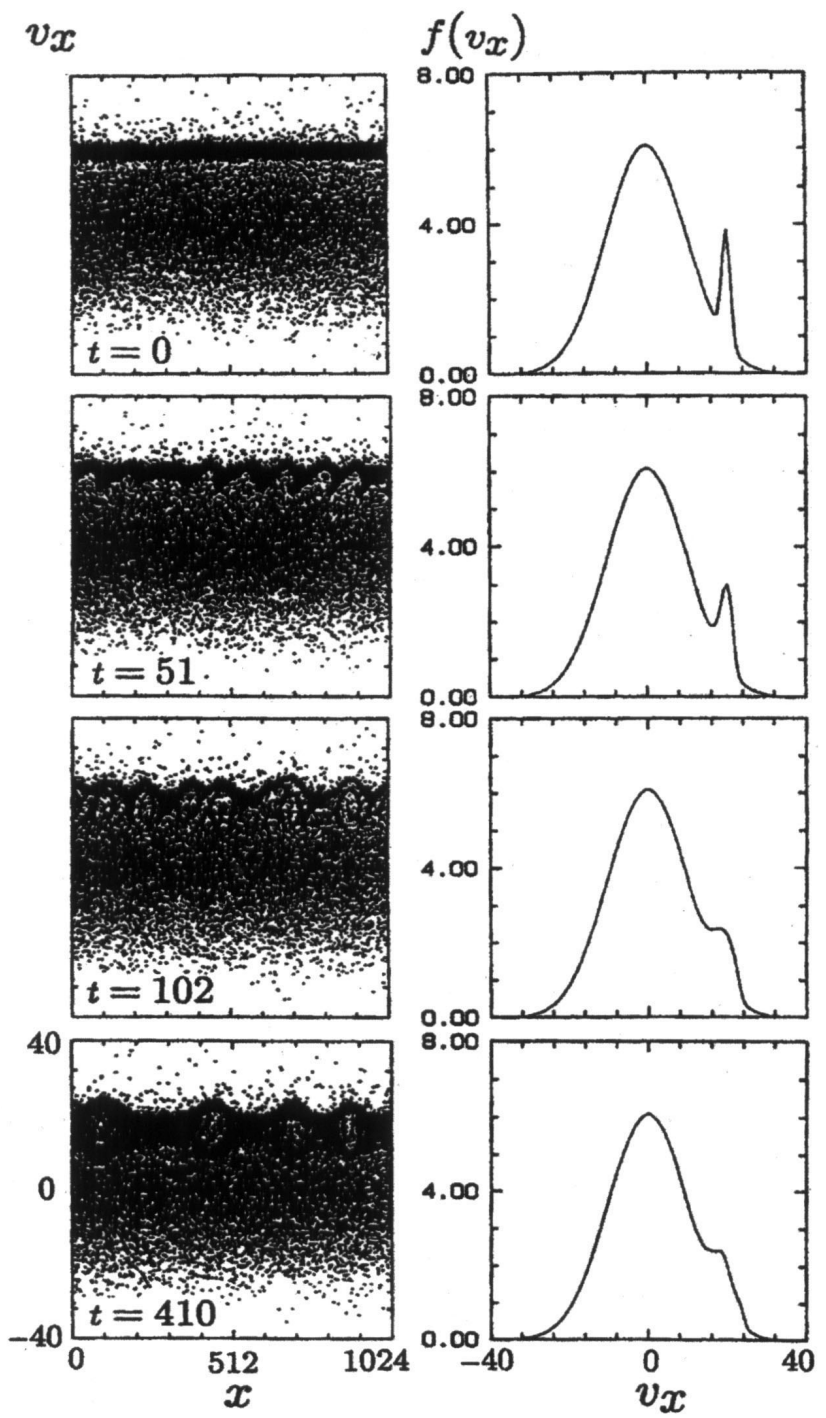

Fig. 5. Time evolution of the bump-on-tail instability. Right and left four panels show the time series of electron phase diagrams and electron velocity distribution (taken from Omura et al., 1996, Fig. 7).

ton models can produce both positive and negative potential structures, depending upon the plasma and field parameters of the region. The 2-D and 3-D particle simulations of the electron beam driven instabilities have given useful information on the life-time of electron holes, as well as on the ion heating (Omura et al., 1999; Goldman et al., 2000; Singh, 2001). The 3-D simulations of electron-beam driven electron holes indicate that, initially a large number of electron holes are formed. They merge continuously, and at a later time, only a few electron holes are left and they decay by emitting low-frequency whistler waves (Singh, 2002). However, only a fraction of the possible parametric space where solitary structure can exist has been simulated. On the theoretical front, there is a need to develop BGK type solutions in 2-D and 3-D for the electrostatic modes and also to extend the analysis to the case of some electromagnetic modes, say,

\section{$|\mathbf{E}(\mathbf{x}, \mathbf{y})|^{2}$}

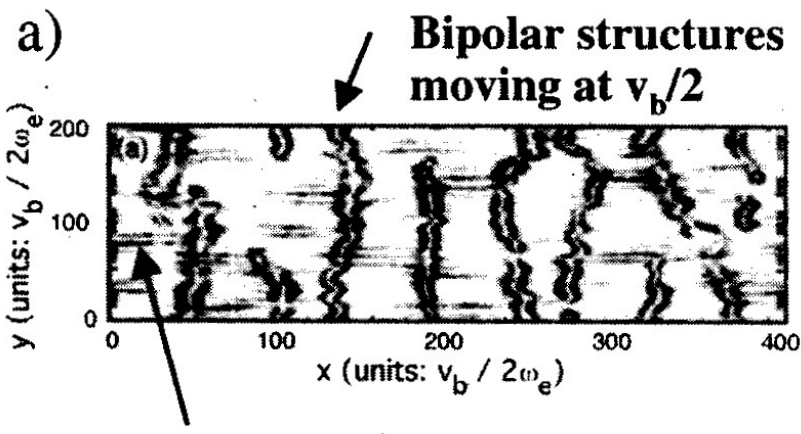

Electrostatic whistlers

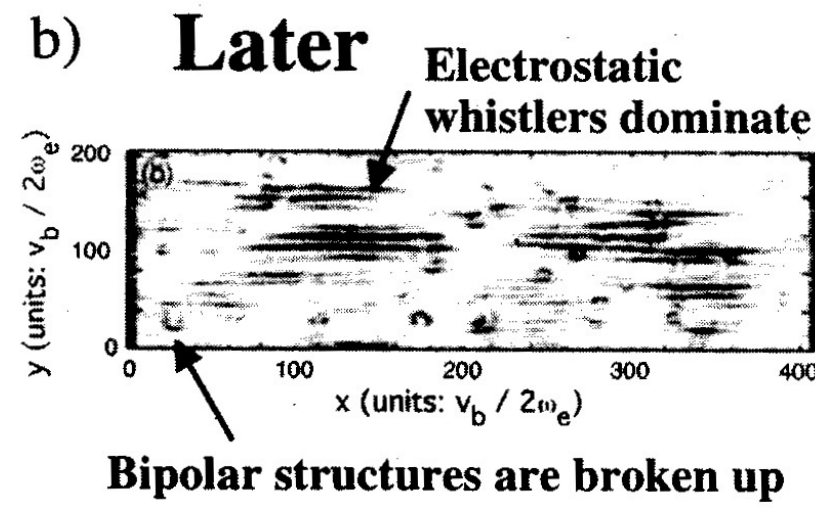

Fig. 6. Wave electric field structures in 2-D. (a) Detail of $|E(x, y)|^{2}$ at an early time showing bipolar structures and low-level whistlers. (b) $|E(x, y)|^{2}$ at late, whistler-dominated time (taken from Goldman et al., 2000).

like whistler, to account for the magnetic component. However, a full kinetic theory would be needed to account for the observed characteristics of the solitary structures.

There is a need to explore the suitability of other soliton models not discussed here, for example, Langmuir, lower hybrid and whistler solitons, for explaining the properties of the observed solitary structures. Polar observations clearly indicate a significant magnetic component associated with the electrostatic bipolar structures (Tsurutani et al., 1998b). A whistler soliton model would be able to explain the magnetic field component associated with the electrostatic solitary pulses in a natural way. The stability of the soliton models in 2-D and 3-D needs to be explored. For example, for 3-D perturbations, LH and whistler solitons are modulationally unstable. This may put restrictions on the possible parameters for where soliton models can be applied to real situations.

Acknowledgements. Portions of this work were performed at the Jet Propulsion Laboratory, California Institute of Technology, 
Electron beam $=8(10 \%) P=5$
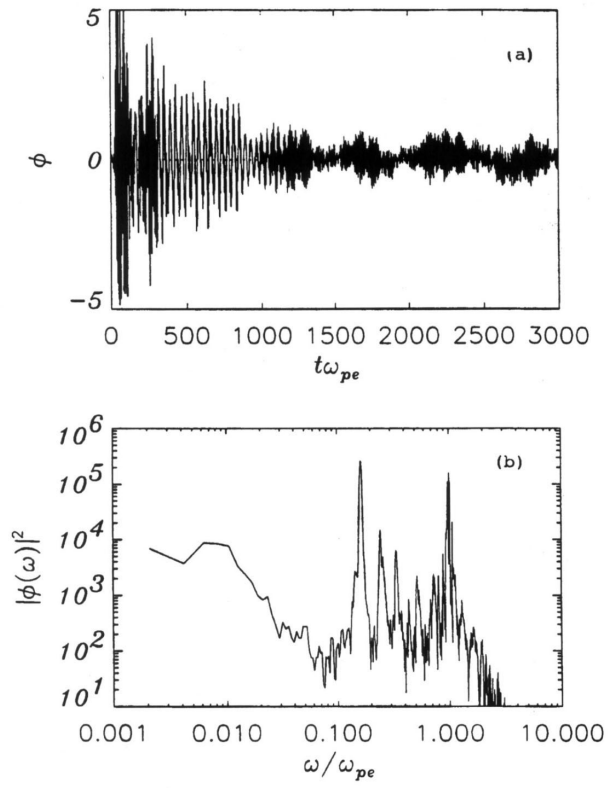

(a)

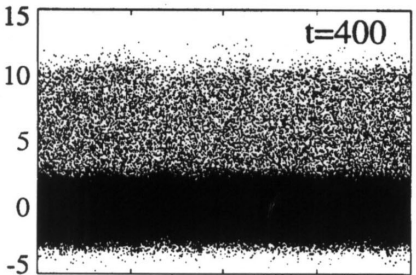

(b)
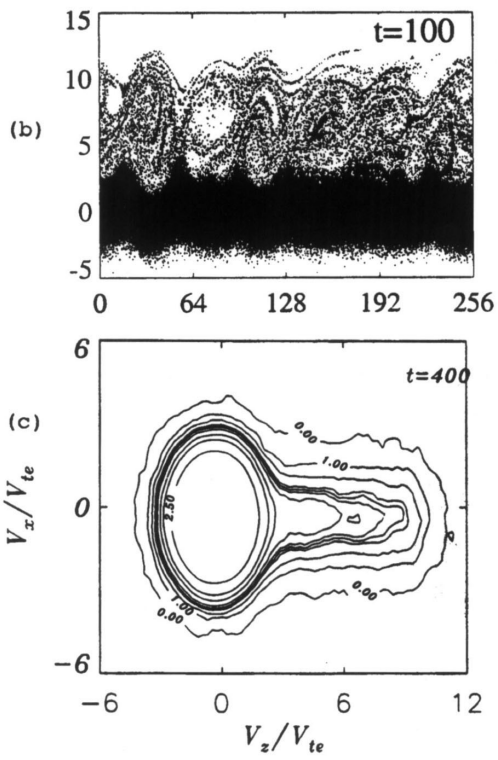

Fig. 7. Left panels show the potential and its Fourier transforms: (a) temporal evolution of the potential at the point P (16, 24, 5). Such a temporal evolution is typical of the entire plasma. (b) The FFT of $\phi(t)$ in panel (a). Right panels show electron phase space and velocity distribution: (b) $V_{z}-V_{x}$ phase space showing vortexes involving the beam electrons at $t=100$. (a) The same phase space at $t=400$, when the vortex structures have dissipated. (c) The velocity distribution $f\left(V_{x}, V_{z}\right)$ is shown by contours of its constant values. The contour levels are on logarithmic scale, and all contours between those labeled with 2.5 and 1 are equispaced with an interval of 0.2 (taken from Singh et al., 2001b).

Pasadena, California, under contract with the National Aeronautics and Space Administration.

\section{References}

Anderson, R. R., Harvey, C. C., Hope, M. M., Tsurutani, B. T., Eastman, T. E., and Etcheto, J.: Plasma Waves Near the Magnetopause, J. Geophys. Res., 87, 2087, 1982.

Bernstein, I. B., Greene, J. M., and Kruskal, M. D.: Exact Nonlinear Plasma Oscillations, Phys. Rev., 108, 546-550, 1957.

Berthomier, M., Pottellette, R., and Malingre, M.: Solitary waves and weak double layers in a two-electron temperature auroral plasma, J. Geophys. Res., 103, 4261, 1998.

Berthomier, M., Pottellette, R., Malingre, M., and Khotyaintsev, Y.: Electron-acoustic solitons in an electron-beam plasma system, Phys. Plasmas, 7, 2987, 2000.

Bharuthram, R. and Shukla, P. K.: Multidimensional solitons and double layers in a magnetized plasma, Phys. Scripta, 34, 732, 1985.

Boström, R., Gustafsson, G., Holback, B., Holmgren, G., Koskinen, H., and Kintner, P.: Characteristics of solitary waves and weak double layers in magnetospheric plasma, Phys. Rev. Lett., 61, 82, 1988.
Bounds, S. R., Pfaff, R. F., Knowlton, S. F., Mozer, F. S., Temerin, M. A., et al.: Solitary potential structures associated with ion and electron beams near $1 R_{E}$ altitude, J. Geophys. Res., 104, 28 709, 1999.

Buti, B.: Ion-acoustic holes in a two-electron temperature plasma, Phys. Lett. 76A, 251, 1980.

Cattell, C., Dombeck, A. J., Wygant, J. R., Hudson, M. K., Mozer, F. S., Temerin, M. A., Peterson, W. K., Kletzing, C. A., Russell, C. T., and Pfaff, R. F.: Comparisons of Polar Satellite Observations of Solitary Wave Velocities in the Plasma Sheet Boundary and the High-altitude Cusp to those in the Auroral Zone, Geophys. Res. Lett., 26, 425, 1999.

Dubouloz, N., Pottelette, R., Malingre, M., and Treumann, R. A.: Generation of Broadband Electrostatic Noise by Electron Acoustic Solitons, Geophys. Res. Lett., 18, 155-158, 1991a.

Dubouloz, N., Pottelette, R., Malingre, M., Holmgren, G., and Lindqvist, P. A.: Detailed Analysis of Broadband Electrostatic Noise in the Dayside Auroral Zone, J. Geophys. Res., 96, 3565, $1991 b$.

Dubouloz, N., Treumann, R. A., Pottelette, R., and Malingre, M.: Turbulence generated by a gas of electron acoustic solitons, J. Geophys. Res., 98, 17 415, 1993.

Ergun, R. E., Carlson, C. W., McFadden, J. P., Mozer, F. S., Delory, G. T., Peria, W., Chaston, C. C., Temerin, M., Elphic, R., 
Strangeway, R., Pfaff, R., Cattell, C. A., Klumpar, D., Shelley, E., Peterson, W., Moebius, E., and Kistler, L.: FAST satellite observations of electric field structures in the auroral zone, Geophys. Res. Lett. 25, 2025-2028, 1998.

Ergun, R. E. et al.: FAST Satellite Observations of LargeAmplitude Solitary Structures, Geophys. Res. Lett., 25, 2041, 1998a.

Ergun, R. E., Carlson, C. W., McFadden, J. P., Mozer, F. S., Muschietti, L., Roth, I., and Strangeway, R.: Debye-Scale Plasma Structures Associated With Magnetic-Field Aligned Electric Fields, Phys. Rev. Lett., 81, 826, 1998 b.

Franz, J. R., Kintner, P. M., and Pickett, J. S.: Polar Observations of Coherent Electric Field Structures, Geophys. Res. Lett., 25, 1277-1280, 1998.

Franz, J. R., Kintner, P. M., Seyler, C. E., Pickett, J. S., and Scudder, J. D.: On the Perpendicular Scale of Electron Phase-Space Holes, Geophys. Res. Lett. 27, 169-172, 2000.

Gendrin, R.: Magnetic turbulence and diffusion processes in the magnetopause boundary layer, Geophys. Res. Lett., 10, 769, 1983.

Ghosh, S. S., Sen, A., and Lakhina, G. S.: Dromion solutions for an electron acoustic wave and its application to space observations, Pramana, 55, 693-698, 2000.

Goldman M. V., Oppenheim, M., and Newman, D. L.: Nonlinear Two-Stream Instabilities as an explanation for the auroral bipolar wave structures, Geophys. Res. Lett., 26, 1821-1824, 1999.

Goldman, M. V., Gary, F., Newman, D. L., and Oppenheim, M.: Turbulence driven by two-stream instability in a magnetized plasma, Phys. Plasmas, 7, 1732, 2000.

Gray, P. C., Hudson, M. K., Lotko, W., and Bergman, R.: Decay of ion beam driven acoustic waves into ion holes, Geophys. Res. Lett., 18, 1675, 1991.

Gurnett, D. A., Anderson, R. R., Tsurutani, B. T., Smith, E. J., Paschmann, G., Haerendel, G., Bame, S. J., and Russell, C. T.: Plasma Wave Instabilities at the Magnetopause: Observations from ISEE 1 and 2, J. Geophys. Res., 84, 7043, 1979.

Hudson, M. K., Lotko, W., Roth, I., and Witt, E.: Solitary waves and double layers on auroral field lines, J. Geophys. Res., 88, 916, 1983.

Kojima, H., Matsumoto, H., Chikuba, S., Horiyama, S., AshourAbdalla, M., and Anderson, R. R.: Geotail Waveform Observations of Broadband/narrowband Electrostatic Noise in the Distant Tail, J. Geophys. Res., 102, 14 439, 1997.

Kojima, H., Omura, Y., Matsumoto, H., Miyaguti, K., and Mukai, T.: Characteristics of Electrostatic Solitary Waves: Statistical Analysis, Nonl. Proc. Geophys., 6, 179, 1999.

Koskinen, H., Lundin, R., and Holback, B.: On the plasma environment of solitary waves and weak double layers, J. Geophys. Res., 95, 5921, 1990.

LaBelle, J. and Treumann, R. A.: Plasma Waves at the Dayside Magnetopause, Space Sci. Revs., 47, 175, 1988.

Lakhina, G. S. and Tsurutani, B. T.: Broadband plasma waves in the magnetopause and polar cap boundary layers, Serv. Geophys., 20, 377, 1999.

Lakhina, G. S., Tsurutani, B. T., Kojima, H., and Matsumoto, H.: Broadband Plasma Waves in the Boundary Layers, J. Geophys. Res., 105, 27 791-27 831, 2000a.

Lakhina, G. S., Tsurutani, B. T., Arballo, J. K., and Galvan, C.: Sun-Earth Connection: Boundary Layer Waves and Auroras, PRAMANA-J. Phys., 55, 665-683, 2000b.

Lee, L. C. and Kan, J. R.: Nonlinear ion-acoustic waves and solitons in a magnetized plasma, Phys. Fluids, 24, 430-433, 1981.
Matsumoto, H., Kojima, H., Miyatake, T., Omura, Y., Okada, M., and Tsutsui, M.: Electrostatic Solitary Waves (ESW) in the Magnetotail - BEN Wave Forms Observed by Geotail, Geophys. Res. Lett., 21, 2915-2918, 1994.

McFadden, J. P., Carlson, C. W., and Ergun, R. E.: Microstructure of the auroral acceleration region as observed by FAST, J. Geophys. Res., 104, 14 453, 1999.

Mozer, F. S., Ergun, R., Temerin, M., Cattell, C., Dombeck, J., and Wygant, J.: New Features of Time Domain Electric-Field Stuctures in the Auroral Acceleration Region, Phys. Rev. Lett. 79, 1281, 1997.

Muschietti, L., Ergun, R. E., Roth, I., and Carlson, C. W.: PhaseSpace Electron Holes along Magnetic Field Lines, Geophys. Res. Lett., 26, 1093, 1999.

Omura, Y., Kojima, H., and Matsumoto, H.: Computer Simulation of Electrostatic Solitary Waves: A Nonlinear Model of Broadband Electrostatic Noise, Geophys. Res. Lett., 21, 2923-2926, 1994.

Omura, Y., Matsumoto, H., Miyake, T., and Kojima, H.: Electron Beam Instabilities as Generation Mechanism of Electrostatic Solitary Waves in the Magnetotail, J. Geophys. Res., 101, 2685, 1996.

Omura, Y., Kojima, H., Miki, N., Mukai, T., Matsumoto, H., and Anderson, R.: Electrostatic Solitary Waves Carried by Diffused Electron Beams Observed by the Geotail Spacecraft, J. Geophys. Res., 104, 14 627, 1999.

Pottelette, R., Malingre, M., Dubouloz, N., Aparicio, B., Lundin, R., Holmgren, G., and Marklund, G.: High Frequency Waves in the Cusp/cleft Regions, J. Geophys. Res., 95, 5957, 1990.

Qian, S., Lotko, W., and Hudson, M. K.: Dynamics of localized ionacoustic waves in a magnetized plasma, Phys. Fluids, 31, 2190, 1988.

Reddy, R. V. and Lakhina, G. S.: Ion-acoustic double layers and solitons in auroral plasma, Planet. Space Sci., 39, 1343, 1991.

Reddy, R. V., Lakhina, G. S., and Verheest, F.: Ion-acoustic double layers and solitons in multispecies auroral beam plasmas, Planet. Space Sci., 40, 1055-1062, 1992.

Reddy, R. V., Lakhina, G. S., Singh, N., and Bharuthram, R.: Spiky parallel electrostatic ion cyclotron and ion acoustic waves, Nonl. Proc. Geophys., 9, 1, 25-29, 2002.

Rezeau, L., Perraut, S., and Roux, A.: Electromagnetic Fluctuations in the Vicinity of the Magnetopause, Geophys. Res. Lett., 13, 1093, 1986.

Singh, N.: Space-time evolution of electron-beam driven electron holes and their effects on the plasma, Nonl. Proc. Geophys., this issue, 2003.

Singh, N., Loo, S. M., Wells, B. E., and Lakhina, G. S.: Evolution of electron beam generated waves resulting into transverse ion heating and filamentation of the plasma, J. Geophys. Res., 106, $21165,2001 b$.

Singh, N., Loo, S. M., and Wells, B. E.: Electron hole structure depending on plasma magnetization, J. Geophys. Res., 106, 21 183, 2001c.

Singh, S. V., Reddy, R. V., and Lakhina, G. S.: Broadband Electrostatic Noise due to Nonlinear Electron Acoustic Waves, Adv. Space Res., 28, 11, 1643, 2001a.

Song, P., Zhu, J., Russell, C. T., Anderson, R. R., Gurnett, D. A., Ogilvie, K. W., and Strangeway, R. J.: Properties of ELF Emission in the Dayside Magnetopause, J. Geophys. Res., 103, 26495, 1998.

Temerin, M., Woldorff, M., and Mozer, F. S.: Nonlinear Steepening of the Electrostatic Ion Cyclotron Wave, Phys. Rev. Lett., 43, 
1941-1943, 1979.

Temerin, M., Cerny, K., Lotko, W., and Mozer, F. S.: Observations of double layers and solitary waves in the auroral plasma, Phys. Rev. Lett., 48, 1175, 1982.

Tsurutani, B. T., Smith, E. J., Thorne, R. M., Anderson, R. R., Gurnett, D. A., Parks, G. K., Lin, C. C., and Russell, C. T.: Wave-Particle Interaction at the Magnetopause: Contribution to the Dayside Aurora, Geophys. Res. Lett., 8, 183, 1981.

Tsurutani, B. T., Brinca, A. L., Smith, E. J., Okida, R. T., Anderson, R. R., and Eastman, T. E.: A Statistical Study of ELF-VLF Plasma Waves at the Magnetopause, J. Geophys. Res., 94, 1270, 1989.

Tsurutani, B. T., Lakhina, G. S., Ho, C. M., Arballo, J. K., Galvan, C., Boonsiriseth, A., Pickett, J. S., Gurnett, D. A., Peterson, W. K., and Thorne, R. M.: Broadband Plasma Waves Observed in the Polar Cap Boundary Layer: Polar, J. Geophys. Res., 103,
17351, 1998a.

Tsurutani, B. T., Arballo, J. K., Lakhina, G. S., Ho, C. M., Buti, B., Pickett, J. S., and Gurnett, D. A.: Plasma Waves in the Dayside Polar Cap Boundary Layer: Bipolar and Monopolar Electric Pulses and Whistler Mode Waves, Geophys. Res. Lett., 25, 4117, $1998 b$.

Turikov, V.: Electron phase space holes as localized BGK solutions, Phys. Scripta, 30, 73, 1984.

Yu, M. Y., Shukla, P. K., and Bujarbarua, S.: Fully nonlinear ionacoustic solitary waves in a magnetized plasma, Phys. Fluids, 23, 2146-2147, 1980.

Zhu, Z., Song, P., Drake, J. F., Russell, C. T., Anderson, R. R., Gurnett, D. A., Ogilvie, K. W., and Fitzenreiter, R. J.: The Relationship between ELF-VLF Waves and Magnetic Shear at the Dayside Magnetopause, Geophys. Res., Lett., 23, 773, 1996. 